Tropical Journal of Pharmaceutical Research, February 2010; 9 (1): 35-43

(C) Pharmacotherapy Group,

Faculty of Pharmacy, University of Benin,

Benin City, 300001 Nigeria.

All rights reserved.

Research Article

Available online at http://www.tjpr.org

\title{
Species Distribution and Antibiotic Resistance in Coagulase-negative Staphylococci Colonizing the Gastrointestinal Tract of Children in Ile-Ife, Nigeria
}

\section{Ezekiel Olugbenga Akinkunmi and Adebayo Lamikanra}

Department of Pharmaceutics, Obafemi Awolowo University, Ile-Ife, Nigeria.

\begin{abstract}
Purpose: The antimicrobial susceptibility of 149 coagulase-negative staphylococci (CoNS) isolates from faecal samples of children in Ile-Ife, Nigeria, was evaluated in order to determine their contribution to antimicrobial resistance in the community.

Methods: The isolates were identified to the species level by conventional methods, and their susceptibility to 20 antibiotics was tested by disk diffusion and to vancomycin by agar dilution.

Results: The species distribution was as follows: Staphylococcus epidermidis 45 (30.2 \%), S. haemolyticus, 26 (17.5\%); S. capitis, 24 (16.1\%); S. xylosus, 11 (7.4\%); S. saprophyticus, 8 (5.4\%); S. warneri, 8 (5.4\%); S. hominis, 6 (4.0\%); S. schleiferi, 5 (3.3\%); S. lugdunensis, $3(2.0 \%)$ and S. capitis sub ureolyticus, $3(2.0 \%)$ and isolates from other CoNS species 10 (6.7\%). Resistance to the $\beta$ lactam antibiotics was in excess of $50 \%$ of the isolates tested whilst there was significant incidence of resistance to cotrimoxazole, chloramphenicol, tetracycline, erythromycin, fusidic acid and norfloxacin. The highest percentage of oxacillin resistance was found among S. haemolyticus (46.2\%) while the lowest was in S. capitis (8.3\%). Reduced susceptibility (MIC $\geq 4 \mathrm{mg} / \mathrm{L}$ ) to vancomycin was shown by both oxacillin-resistant and susceptible CoNS species.

Conclusion: The gastrointestinal tracts of children could serve as a reservoir for antibiotic-resistant CoNS, some of which had reduced susceptibility to vancomycin.
\end{abstract}

Keywords: Coagulase-negative staphylococci, gastrointestinal tract, antibiotics resistance, vancomycin resistance, children, Nigeria. 


\section{INTRODUCTION}

The gastrointestinal tract has been recognised as a major ecological site for various bacteria that can reach neighbouring sterile sites and cause mild infections or invasive diseases [1]. Recent data suggest that the human gastrointestinal tract may be an important reservoir of multiple antibioticresistant $S$. aureus strains and there is considerable evidence that the gastrointestinal tract also provides an important source for transmission and dissemination of these organisms [2].

So far, all studies investigating staphylococci carriage in the intestine, except the two reported by Domniguez et al [3] on Spanish children and by Meisel-Mikolajczyk et al., [4] on Polish neonates, have specifically looked for $S$. aureus. This is not surprising since $S$. aureus, a coagulase-positive staphylococcal species, being the most pathogenic species of the staphylococci, is one of the most common causes of both community and hospital-acquired infections of skin, surgical site, blood and the lower respiratory tract [5]. However, coagulase-negative staphylococci (CoNS) species, which used to be found as contaminants of blood cultures, are also increasingly associated with a variety of infections [6] and thus should not be overlooked.

Although $S$. epidermidis is the CoNS most commonly isolated in the clinical microbiology laboratory, other CoNS species are also increasingly associated with infections [7]. For example, $S$. haemolyticus plays an important role in hospital-acquired opportunistic infections. It is second only to $S$. epidermidis in its frequency of isolation from human blood cultures and has been reported to cause peritonitis, otitis media, urinary tract infections and septicemia. $S$. saprophyticus is the second most common pathogen identified in acute uncomplicated urinary tract infections.
In addition, S. lugdunensis causes infections resembling those caused by $S$. aureus rather than those caused by its coagulase-negative staphylococcal counterparts. Other clinically significant species of CoNS include; $S$. hominis, $S$. warneri, $S$. capitis, $S$. saccharolyticus, S. auricularis, S. caprae, and S. schleiferi [7].

The clinical significance of CoNS strains is also underscored by their resistance characteristics. CoNS strains have acquired resistance to methicillin, and majority of them are also resistant to almost all classes of antimicrobial agents [8]. Because of high oxacillin resistance rates among CoNS, vancomycin is the antimicrobial agent of choice. However, a trend toward decreased susceptibility to this glycopeptide has been shown among CoNS isolates [8]. Limited treatment options and prolonged course of infection due to these CoNS species could, therefore, have severe consequences.

Because of the increasing clinical significance of CoNS, accurate species identification of CoNS is highly desirable to permit a more precise determination of hostpathogen relationship of CoNS and to establish epidemiological trends. This study investigates the carriage of CoNS in the gastrointestinal tract of children under the age of 3 years in lle-Ife, Nigeria and describes the isolation of antibiotic-resistant and clinically significant species of CoNS from faecal samples of the children.

\section{EXPERIMENTAL}

\section{Study area and population}

The study was carried out in Ile-Ife, a semiurban town in Osun State of Nigeria and the adjoining villages. The study focused on children of both sexes under 3 years of age. Subjects were drawn from children attending immunisation clinics and children at day care centres in the community and from households selected randomly within lle-lfe. All subjects were not reported to have taken 
any antimicrobial agent in the last two weeks preceding sampling. The study was approved by the institutional review boards of each of the participating institutions and all specimens were collected with the informed consent of the parents or guardians of the children.

\section{Sample collection and phenotypic identification of isolates}

Freshly voided stools were collected over a period of six months (January to June, 2006) from a total of 293 children of which 165 $(56.3 \%)$ were male and $128(43.7 \%)$ female. The specimens collected were plated on mannitol salt agar and incubated at $37{ }^{\circ} \mathrm{C}$ for 24 - 48 hours. Pure colonies obtained were then subcultured onto fresh mannitol salt agar and blood agar. The presence or absence of growth, colour, average colony size, margin, surface elevation, opacity, and consistency of colonies produced by each isolate on the media was noted. CoNS strains were identified by these colonial characteristics and by Gram stain reactions (Gram-positive cocci in bunches), positive catalase test, negative modified oxidase test, and negative result to the tube coagulase test. The method described by leven et al., [9] was further employed in the biochemical characterisation of the CoNS including alkaline phosphatase test, fermentation of threhalose, xylose and mannitol, growth in thioglycollate broth and susceptibility to novobiocin. The isolates were cryopreserved in cryovials (Nalgene, Rochester, NY and USA) and stored at $-20^{\circ} \mathrm{C}$.

\section{Molecular confirmation of CoNS}

PCR assays to detect the nuc gene were conducted to confirm phenotypic identification of the isolates as coagulase-negative staphylococci as described previously [10]. The primers used were nuc- $F$ (5'GCGATTGATGGTGATACGGTT-3') and nuc-R (5'-AGCCAAGCCTTGACGAACTAAA GC-3') as described [10].
PCR conditions for nuc gene comprised a predenaturation step of $95^{\circ} \mathrm{C}$ for 5 minutes, followed by 40 cycles of denaturation at $95^{\circ} \mathrm{C}$ for 30 seconds, annealing at $55^{\circ} \mathrm{C}$ for 30 seconds, and extension at $72^{\circ} \mathrm{C}$ for 1 minute, followed by final extension for 5 minutes at $72^{\circ} \mathrm{C}$.

The samples were electrophoresed for 40 min at 90V. A 100-bp DNA Molecular Weight Marker XIV (Roche Diagnostics $\mathrm{GmbH}$, Germany) was used as a molecular weight marker in each gel. DNA fragments of $280 \mathrm{bp}$ which corresponded to the nuc PCR product were visualized for Staphylococcus aureus strains which were used as positive control but were absent for all the CoNS strains screened using a UV transilluminator at $300 \mathrm{~nm}$ after staining with ethidium bromide for 15 minutes and destaining for 30 minutes.

\section{Antibiotic sensitivity test}

The standard disc diffusion method approved by the Clinical Laboratory Standard Institute (CLSI) [11] was employed. The antibiotic discs employed were produced by Abtek, England; AB-Biodisc, Sweden and Oxoid, England. The following antimicrobial agents at the indicated concentration were tested: Clindamycin (CM) $15 \mu \mathrm{g} /$ disc, chloramphenicol (CL) $30 \mu \mathrm{g} /$ disc, trimethoprim (TR) 5 $\mu \mathrm{g} / \mathrm{disc}$, cephalothin (CE) $30 \mu \mathrm{g} /$ disc, fusidic acid (FU) $50 \mu \mathrm{g} /$ disc, ciprofloxacin (Cl) 10 $\mu \mathrm{g} / \mathrm{disc}$, piperacillin (PP) $30 \mu \mathrm{g} / \mathrm{disc}$, amoxicillin (AMX) $25 \mu \mathrm{g} /$ disc, norfloxacin (NX) $10 \mu \mathrm{g} /$ disc, penicillin V (PV) $10 \mu \mathrm{g} / \mathrm{disc}$, cephadroxil (DX) $30 \mu \mathrm{g} /$ disc, erythromycin (EM) $15 \mu \mathrm{g} /$ disc, tobramycin (TM) $30 \mu \mathrm{g} / \mathrm{disc}$, sulfisoxazole (SU) $250 \mu \mathrm{g} /$ disc, vancomycin (VA) $5 \mu \mathrm{g} /$ disc, methicillin (ME) $10 \mu \mathrm{g} /$ disc, by AB-Biodisc; amikacin (AKN) $10 \mu \mathrm{g} /$ disc by Institute Pasteur; tetracycline (TE) $30 \mu \mathrm{g} /$ disc, co-trimoxazole (COT) $25 \mu \mathrm{g} / \mathrm{disc}$, gentamicin (GEN) $30 \mu \mathrm{g} /$ disc, augmentin (AUG) 30 $\mu \mathrm{g} / \mathrm{disc}$, cloxacillin (CXC) $5 \mu \mathrm{g} / \mathrm{disc}$ by Abtek, and streptomycin (STR) $30 \mu \mathrm{g} /$ disc, ampicillin (AMP) $10 \mu \mathrm{g} /$ disc, oxacillin (OX) $1 \mu \mathrm{g} /$ disc by Oxoid. The tests were carried out on Mueller- 
Hinton agar (Remel, England) following the protocol given by CLSI [11].

\section{Determination of minimum inhibitory concentration (MIC) of vancomycin}

Several dilutions of vancomycin (Sigma Chemical Company, U.S.A.) were prepared from the powder to give plates with final concentrations of antimicrobial incorporated of 32, $168,4 \mathrm{mg} / \mathrm{L}, 2$ and $1 \mathrm{mg} / \mathrm{L}$. An 0.5 McFarland inoculum of each isolate whose MIC was to be determined was used. Inoculation of the vancomycin-agar plates was performed with a multipoint inoculator. If any growth occurred within $48 \mathrm{~h}$, the isolate was considered to be vancomycin resistant. The experiment was performed in duplicate. CLSI [11] guidelines were used for vancomycin MIC breakpoints of susceptible (less than $4 \mathrm{mg} / \mathrm{L}$ ), intermediate (4 to 8 $\mathrm{mg} / \mathrm{L})$, and resistant (16mg/L or more).

\section{Statistical Analysis}

Chi-square test or the Fisher exact test was used in determining probabilities and level of significance. All hypotheses were considered significant if $p<0.05$.

\section{RESULTS}

\section{Carriage of CoNS strains by the children}

Of the 293 children, 118 (40.3\%) were colonised by various species of CoNS (see Table 1). Several children were carriers of more than one species of CoNS. Twenty five $(8.5 \%)$ and $2(0.7 \%)$ had two and three species of CoNS isolated from them, respectively. Of the 2 children with three species of CoNS isolated, one was a 20-day old male child with $S$. capitis, $S$. xylosus and $S$. saprophyticus isolated while the other was an 18-month old female child with $S$. capitis, $S$. xylosus and $S$. warneri isolated. None of the subjects had more than three different species isolated from them.
A significantly greater number of $S$. epidermidis was isolated from male $(73.3 \%)$ than female $(26.7 \%)$ children $(p<0.05)$. For the other CoNS species, there was no significant relationship between faecal carriage and children characteristics such as the age and gender of the children $(p>0.05)$.

Table 1: Species distribution of CoNS isolated from the faecal samples of children

\begin{tabular}{lll}
\hline CoNS strain & Frequency & $\%$ \\
\hline S. epidermidis & 45 & 30.2 \\
S. haemolyticus & 26 & 17.5 \\
S. capitis & 24 & 16.1 \\
S. xylosus & 11 & 7.4 \\
S. saprophyticus & 8 & 5.4 \\
S. warneri & 8 & 5.4 \\
S. hominis & 6 & 4.0 \\
S. schleiferi & 5 & 3.3 \\
S. lugdunensis & 3 & 2.0 \\
S. capitis sub & 3 & 2.0 \\
ureolyticus & 10 & 6.7 \\
Unspeciated CoNS & & \\
\multicolumn{1}{c}{ Total } & 149 & 100.0 \\
\hline
\end{tabular}

\section{Antibiotics resistance in CoNS}

Table $2 \mathrm{a}$ and $2 \mathrm{~b}$ show the antibiotic resistance profiles of the CoNS strains isolated. CoNS isolates that were simultaneously resistant to more than four antibiotics were regarded as being multiply resistant and $92.5 \%, 94.7 \%$, and $100 \%$ of $S$. epidermidis, $S$. capitis and $S$. haemolyticus, respectively, were found to be multiply resistant. Figure 1 shows the antibiotic resistance in methicillin-resistant CoNS (MRCoNS) and methicillin-susceptible CoNS (MSCoNS) isolates. Most of the oxacillinresistant isolates were also found to be resistant to other agents except for cotrimoxazole and ciprofloxacin 
Akinkunmi \& Lamikanra

Table 2a: Antibiotic resistance profile of the individual CoNS species isolated from faecal samples of children

\begin{tabular}{|c|c|c|c|c|c|c|c|c|c|c|c|c|}
\hline \multirow[b]{2}{*}{ Organism (no.) } & \multirow[b]{2}{*}{$\mathrm{CM}$} & \multirow[b]{2}{*}{ TE } & \multirow[b]{2}{*}{ СOT } & \multicolumn{5}{|c|}{ Antibiotic resistance, no. (\%) } & \multirow[b]{2}{*}{ FU } & \multirow[b]{2}{*}{$\mathrm{Cl}$} & \multirow[b]{2}{*}{ PP } & \multirow[b]{2}{*}{ NX } \\
\hline & & & & CL & GEN & TR & AUG & CE & & & & \\
\hline S. epidermidis (45) & $1(2.2)$ & $11(24.4)$ & 15 (33.3) & $13(28.9)$ & $4(8.9)$ & $27(60.0)$ & $23(51.1)$ & $30(66.6)$ & $9(20.0)$ & $5(11.1)$ & $37(82.2)$ & $11(24.4)$ \\
\hline S. capitis (24) & $2(8.3)$ & $6(25.0)$ & $9(37.5)$ & $7(29.1)$ & $2(8.3)$ & $6(25.0)$ & $14(58.3)$ & $12(50.0)$ & $7(29.2)$ & $4(16.7)$ & $14(58.3)$ & $6(25.0)$ \\
\hline S. haemolyticus (26) & $3(11.5)$ & $8(30.8)$ & $16(61.5)$ & $11(42.3)$ & $3(11.5)$ & $7(26.9)$ & $21(80.8)$ & $20(76.9)$ & $6(23.1)$ & $4(15.3)$ & $19(73.1)$ & $8(30.8)$ \\
\hline S. xylosus (11) & $0(0.0)$ & $5(45.5)$ & $8(72.7)$ & $6(54.5)$ & $1(9.1)$ & $5(45.5)$ & $10(90.9)$ & $9(81.9)$ & $5(45.5)$ & $2(18.2)$ & $8(72.7)$ & $4(36.4)$ \\
\hline Other CoNS (43) & $1(2.3)$ & $21(48.8)$ & $22(51.2)$ & 14 (32.6) & $3(7.0)$ & $16(37.2)$ & $25(58.1)$ & $32(74.4)$ & $21(48.8)$ & $4(9.3)$ & $29(67.4)$ & $16(37.2)$ \\
\hline Total (149) & $7(4.7)$ & $51(34.2)$ & $70(47.0)$ & $51(34.2)$ & $13(8.7)$ & $61(40.9)$ & $93(62.4)$ & $103(69.1)$ & 48 (32.2) & $19(12.8)$ & $107(71.8)$ & $45(30.2)$ \\
\hline
\end{tabular}

CM: Clindamycin; TE: Tetracycline; COT: Cotrimoxazole; CL: Chloramphenicol; GEN: Gentamicin; TR: Trimethoprim; AUG: Augmentin; CE: Cephalothin; FU: Fusidic acid; Cl: Ciprofloxacin; PP: Piperacillin; NX: Norfloxacin 
Table 2b: Antibiotic resistance profile of the individual CoNS species isolated from faecal samples of children

\begin{tabular}{lllllllll}
\hline \multicolumn{7}{c}{ Antibiotic resistance, no. (\%) } \\
Organism (no.) & PV & DX & ERY & TM & AKN & SU & VA & OX \\
\hline $\begin{array}{l}\text { S. epidermidis } \\
\text { (45) }\end{array}$ & $35(77.8)$ & $26(57.8)$ & $17(37.8)$ & $10(22.2)$ & $7(15.6)$ & $17(37.8)$ & $1(2.2)$ & $16(35.6)$ \\
$\begin{array}{l}\text { S. capitis (24) } \\
\text { S. haemolyticus }\end{array}$ & $16(66.7)$ & $14(58.4)$ & $9(37.5)$ & $5(20.8)$ & $3(12.6)$ & $11(45.8)$ & $3(12.5)$ & $2(8.3)$ \\
$\begin{array}{l}\text { (26) } \\
\text { S. xylosus (11) }\end{array}$ & $11(100.0)$ & $10(90.9)$ & $7(63.7)$ & $2(18.2)$ & $0(0.0)$ & $7(63.6)$ & $3(27.3)$ & $3(27.3)$ \\
$\begin{array}{l}\text { Other CoNS } \\
(43)\end{array}$ & $32(74.4)$ & $31(72.1)$ & $21(48.8)$ & $10(23.3)$ & $5(11.6)$ & $19(44.2)$ & $6(14.0)$ & $12(27.9)$ \\
Total (149) & $116(77.9)$ & $99(66.4)$ & $68(45.6)$ & $33(22.1)$ & $21(14.1)$ & $68(45.6)$ & $17(11.4)$ & $45(30.2)$ \\
\hline
\end{tabular}

PV: Penicillin V; DX: Cephadroxil; ERY: Erythromycin; TM: Tobramycin; AKN: Amikacin; SU: Sulfisoxazole; VA: Vancomycin; OX: Oxacillin

\section{Carriage of MRCoNS by children}

Of the 149 CoNS isolated from the children $45(30.2 \%)$ were found to be MRCoNS.

\section{MIC of vancomycin}

Eight MRCoNS and 9 MSCoNS isolates showed MIC $\geq 4 \mathrm{mg} / \mathrm{L}$ and were, therefore, considered to have shown reduced susceptibility to vancomycin. Of the eight MRCoNS, 1 each was $S$. epidermidis, $S$. warneri, S. lugdunensis and S. xylosus and 2 each were $S$. capitis and $S$. haemolyticus while the MSCoNS were 1 each of $S$. capitis and 2 each of $S$. haemolyticus, S. xylosus, $S$. hominis and the unspeciated CoNS.

\section{DISCUSSION}

Many laboratories do not identify clinical isolates of CoNS to the species level as they are considered normal inhabitants of skin and nares capable of causing only opportunistic infections. However, the increase in the implication of CoNS as significant nosocomial pathogens with a high rate of resistance to antimicrobial agents has underlined the need for species identification which is important in monitoring the reservoir and distribution of CoNS involved in infections and determining the aetiological agent [6].

Various authors have observed variability in species distributions of CoNS and in their antibiotic susceptibility patterns probably reflecting different protocols of investigations and panels of antibiotics used in different hospitals as well as differences in the geographical locations from where these isolates were obtained [12].

In this study, phenotypic and genotypic characteristics were used in the identification of the CoNS isolates. The absence of the $S$. aureus specific nuc gene supported the phenotypic identification of the isolates as CoNS.

A total of $118(40.3 \%)$ of the subjects were colonized with the CoNS which comprised 149 isolates. The species of CoNS isolated in this study were slightly different from those isolated by Domniguez et al from Spanish children [3]. While those workers isolated 15 


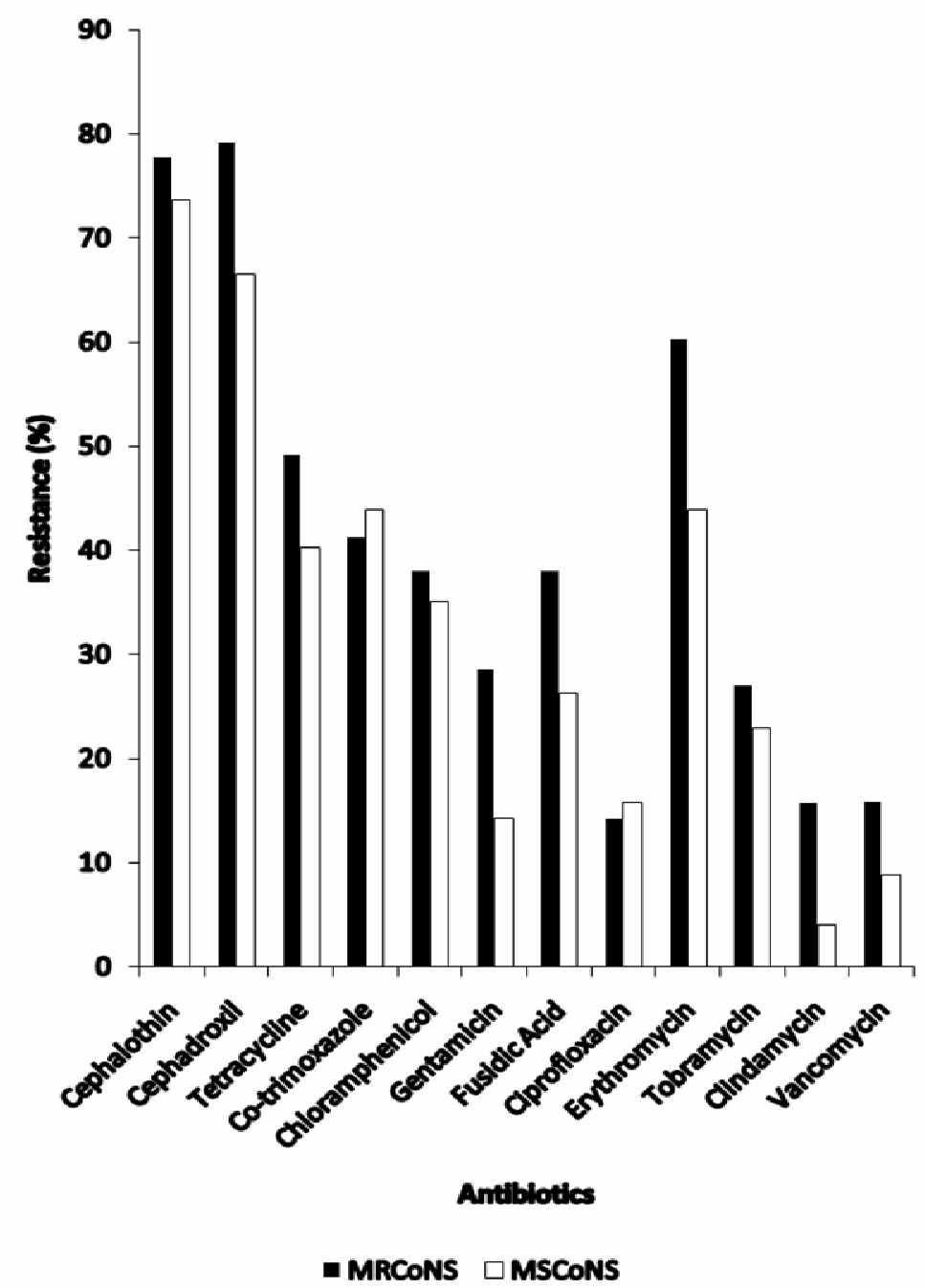

Figure 1: Antibiotic resistance in methicillin-resistant CoNS (MRCoNS) and methicillin-susceptible CoNS (MSCoNS) isolates

S. epidermidis (41.7\%), 8 S. simulans $(22.2$ $\%), 5$ S. xylosus $(13.9 \%), 3$ S. warneri $(8.3$ $\%), 2$ S. haemolyticus $(5.6 \%), 2$ S. capitis $(5.6 \%)$ and $1 S$. caprae $(2.8 \%)$, isolates in this study included $45 \mathrm{~S}$. epidermidis $(30.2$ $\%), 26$ S. haemolyticus, (17.5\%); 24 S. capitis, $(16.1 \%) ; 11$ S. xylosus, $(7.4 \%) ; 8$ S. saprophyticus, (5.4\%); 8 S. warneri, (5.4\%); 6 S. hominis, (4.0\%); 5 S. schleiferi, (3.3\%); 3 S. lugdunensis, $(2.0 \%)$ and 3 S. capitis sub ureolyticus, $(2.0 \%)$ and 10 unspeciated CoNS $(6.7 \%)$. The difference in the rate of isolation of different species of CoNS in this study when compared with the report of Domniguez et al is not unexpected [3]. It suggests that carriage of CoNS species in the faecal samples of children may differ from one country to another.

S. epidermidis was the CoNS isolate with the highest frequency in this study and this is consistent with the reports of its isolation from clinical samples documented in various published studies $[3,12]$. CoNS species that 
were not previously reported to be isolated from faecal samples including $S$. saprophyticus, S. lugdunensis, S. schleiferi and $S$. capitis sub ureolyticus were isolated. These organisms have been documented as established pathogens with a high rate of resistance to antibiotics [8]. The result of this study also showed incidence of colonisation of some children with more than one species of CoNS. CoNS strains isolated from the faecal samples of children were assayed for susceptibility to a range of commonly and uncommonly used antibiotics in the study area. The result showed that the CoNS isolates had considerable resistance to many of the antibiotics against which they were tested.

Penicillin resistance in CoNS isolates ranged from $66.7 \%$ in S. capitis to $100.0 \%$ in S. xylosus. The rate of penicillin resistance was comparable with that obtained by Domniguez et al [3] (83.3\%) in healthy children and by Mohan et al [12] (90.6\%) in clinical isolates of CoNS. Other $\beta$-lactam antibiotics screened generally showed a high resistance rate (greater than $50 \%$ ) to all the CoNS species and may, therefore, not be useful for the therapy of infections caused by these organisms.

In this study, 45 (30.2\%) of the 149 CoNS isolates were methicillin-resistant. There was reduced susceptibility to most antibiotics by MRCoNS isolates compared with MSCoNS strains. It may, therefore, be difficult to find antibiotics to use in treating infections caused by these organisms. However, most (more than $80 \%$ ) of the species of CoNS were inhibited by amikacin, ciprofloxacin, clindamycin, gentamicin and vancomycin. Hence, it may be prudent to use these agents for empirical treatment of serious infections in hospitalised patients. The higher resistance rate shown by the quinolone, norfloxacin (30.2\%), compared with ciprofloxacin (12.8 $\%)$ against the isolates is not surprising since intrinsically, it has a comparably less potency in vitro [13].
Resistance rate to ciprofloxacin (12.8\%) obtained in this study was low when compared with the $51 \%$ obtained by Mohan et al [12] in clinical CoNS isolates but was very much higher than that obtained by Domniguez et al which was $2.6 \%$ [3]. The latter workers, on the other hand, had a higher resistance rate for tobramycin (36\%) and erythromycin (64\%) compared with 22.1 and $45.6 \%$, respectively, obtained in this study.

The rate of tetracycline resistance (34.2\%) was also higher than that obtained by Domniguez et al (20.5 \%) [3]. Despite the fact that tetracycline is not recommended for children, this high rate of resistance is not surprising because tetracycline is one of the antibiotics that has been seriously abused by over-use in the study area [14].

Resistance to cotrimoxazole (trimethoprimsulphamethoxazole) (47\%) is comparable to that of sulfisoxazole (45.6\%). However, resistance rate to trimethoprim in CoNS isolates was shown to be comparably lower $(40.9 \%)$. S. epidermidis showed a considerably higher $(60.0 \%)$ resistance rate to trimethoprim compared with other less common CoNS species (25.0 to $45.5 \%$ ). However, the resistance rate of $S$. epidermidis to cotrimoxazole was $33.3 \%$, considerably lower than the values obtained for other CoNS species.

Evidence indicates increasing tendency towards decreasing susceptibility to vancomycin and other glycopeptides in CoNS species worldwide [15] and the result of this study is in agreement with these observations.

\section{CONCLUSION}

The study suggests that children in lle-lfe harbour multiply antibiotic-resistant CoNS strains in their intestines. This suggests the need for appropriate antimicrobial use to halt or at least limit the spread of resistance. The determination of the species of CoNS could 
help in determining the contribution of each species to antibiotic resistance in the community and help in designing effective surveillance and control strategies. The prevention of or therapy for intestinal carriage of CoNS should also be clinically beneficial. More comprehensive surveillance studies should be performed to obtain updated information about the carriage rate and antimicrobial resistance of these organisms in the intestinal tract of children.

\section{ACKNOWLEDGMENT}

We thank the management and staff of the institutions where samples were collected for support and technical assistance, the International Program in the Chemical Sciences for some antibiotic discs, and Dr. Iruka Okeke of Haverford College, USA, for the provision of all materials for molecular studies and for helpful discussions.

\section{REFERENCES}

1. Leszczynsk $P$, Weber-Dabrowska $B$, Kohutnicka $M$, Luczak M, Gorecki A, Gorski A. Successful eradication of Methicillin Resistant Staphylococcus aureus (MRSA) intestinal carrier status in a Heathcare Worker - Case Report. Folia Microbiology 2006; 51(3): 236238.

2. Boyce JM, Havill NL, Otter JA, Adams NM. Widespread environmental contamination associated with patients with diarrhea and methicillin-resistant Staphylococcus aureus colonization of the gastrointestinal tract. Infect Control Hosp Epidemiol 2007; 28: 1142-1147.

3. Domínguez E, Zarazaga M, Torres C. Antibiotic Resistance in Staphylococcus Isolates Obtained from Fecal Samples of Healthy Children. J. Clin. Microbiol. 2002; 40:26382641.
4. Meisel-Miko,ajczyk F, Rafa,owska K, K.dzielska J, Balcerzak A, Szcz«sny A, Broæ M, KlajdaNowak R. Preliminary studies of infant fecal flora. Med Sci Monit 1997; 3(6): 789-794.

5. Lowy FD. Staphylococcus aureus infections. $N$. Engl. J. Med. 1998; 339: 520-532.

6. Huebner J, Goldmann DA. Coagulase-negative staphylococci: role as pathogens. Annu. Rev. Med. 1999; 50: 223-236.

7. Delmas J, Chacornac JP, Robin F, Giammarinaro $P$, Talon R, Bonnet $R$. Evaluation of the Vitek 2 System with a Variety of Staphylococcus Species. J. Clin Microbiol 2008; 46(1): 311313.

8. Archer GL, Climo MW. Antimicrobial susceptibility of coagulase-negative staphylococci. Antimicrob Agents Chemother 1994; 38: 2231-2237.

9. leven $M$, Verhoeven J, Pattyn SR, Goossens $H$. Rapid and economical method for species identification of clinically significant coagulasenegative staphylococci. J. Clin. Microbiol. 1999; 33: 1060-1063.

10. Brakstad OG, Aasbakk K, Maeland JA. Detection of Staphylococcus aureus by polymerase chain reaction amplification of the nuc gene. Journal of Clinical Microbiology 1992; 30: 1654-1660.

11. Clinical and Laboratory Standards Institute. Performance standards for antimicrobial susceptibility testing; 16th informational supplement. M100-S16. Clinical and Laboratory Standards Institute, Wayne, Pa., 2006.

12. Mohan $U$, Jindal $N$, Aggarwal $P$. Species distribution and antibiotic sensitivity pattern of coagulase negative staphylococci isolated from various clinical specimens. Indian J Med Microbiol 2002; 20: 45-46.

13. Sweetman SC, ed. Martindale, The Complete Drug Reference. Pharmaceutical Press, London. 2005; pp 238-239.

14. Okeke IN, Lamikanra $A$, Edelman $R$. Socioeconomic and behavorial factors leading to acquired bacterial resistance to antibiotics in developing countries. Emerg Infect Dis 1999; 5: 18-27.

15. Center KJ, Reboli AC, Hubler R, Rodgers GL, Long SS. Decreased vancomycin susceptibility of coagulase-negative staphylococci in a neonatal intensive care unit: evidence of spread of Staphylococcus warneri. J. Clin. Microbiol. 2003; 41: 4660-4665. 